\title{
Vector-borne disease, climate change and urban design
}

\author{
Ogden $\mathrm{NH}^{1 *}$
}

\begin{abstract}
Affiliation
${ }^{1}$ National Microbiology Laboratory, Public Health Agency of Canada, Saint-Hyacinthe, QC

*Correspondence: nicholas.ogden@canada.ca
\end{abstract}

Suggested citation: Ogden NH. Vector-borne disease, climate change and urban design. Can Comm Dis Rep 2016;42:202. https://doi.org/10.14745/ ccdr.v42i10a04

\section{Background}

Since the scientific and public health communities began to consider the health effects of climate change, the risk of vector-borne diseases (VBDs) have been at the forefront of discussions because of the sensitivity of mosquitoes and ticks, and the diseases they transmit, to climate and climate change (1).

\section{Objective}

To review the risks of emergence and re-emergence of tick- and mosquito-borne diseases in Canada, and propose that urban and suburban planning and design may modulate these risks.

\section{Narrative}

Mosquitoes and ticks are susceptible to climate because a) their mortality rates are affected by temperature and humidity; b) the rates of progress from one life stage to the next (and thus their lifecycle length and abundance) are temperature-dependent; c) breeding rates are affected by climate; and d) host-seeking activity of vectors varies with temperature and humidity. For mosquito-borne diseases the extrinsic incubation period - or the time it takes for a virus to get from the gut of a mosquito to its salivary glands - depends on temperature. If the climate is too cold, the extrinsic incubation period is longer than the average survival time of a mosquito so viral transmission will not occur. However, different VBDs will respond differently to the long-term changes in temperature and rainfall patterns and the increased climate variability and extreme weather events that are expected to occur with climate change.

New mosquito-borne and tick-borne pathogens may emerge and spread in Canada as climate change makes more of the country suitable for the vectors and pathogens. However, these may follow different patterns depending on the vector. Variable climate and extreme weather events can result in epidemics of mosquito-borne diseases but not of tick-borne diseases because of the vectors' different lifecycles (2). This has been shown by the epidemiologic patterns of West Nile virus, which are characterized by initial rapid geographic spread followed by endemicity and a decline in activity, but with occasional epidemics in some years when rainfall and temperature patterns drive vector abundance. However, the emergence of Lyme disease has been characterized by slow geographic spread followed by relatively constant levels of risk year-on-year. Model-based risk assessments of the emergence of Lyme disease in Canada suggest a geographically widespread future risk; to date, these have been consistent with recent surveillance. Risk assessments for more tropical mosquito-borne diseases such as Zika and chikungunya suggest that risk will increase but will likely be limited to certain areas in southern Canada.

The actual risk posed to the Canadian public by current trends in VBDs may be modulated by urban and suburban planning. Heavy rainfall is more likely to increase mosquito breeding in suburban areas, but drought allows stagnant pools of water in drains and sewers in urban areas to act as breeding grounds (3). Suburban landscape design will determine the degree to which residents come into contact with disease vectors: the greening' of urban spaces to reduce heat islands combined with public health encouragement to walk and cycle may increase our contact with mosquitoes and ticks. Suburban gardens have few species of birds and mosquitoes - but those that are present are often efficient at carrying and transmitting West Nile virus, thus increasing the risk of West Nile viral infection.

\section{Conclusion}

Climate change is likely to drive the emergence and re-emergence of VBDs in Canada (4). However, the degree to which the Canadian public will be at risk from these diseases will be determined, at least in part, by how we design, build and manage urban and suburban environments.

\section{Conflict of interest}

None.

\section{References}

1. Githeko AK, Lindsay SW, Confalonieri UE, Patz JA. Climate change and vector-borne diseases: a regional analysis. Bull World Health Organ. 2000;78:1136-47.

2. Ogden NH, Lindsay LR. 2016. Effects of climate and climate change on vectors and vector-borne diseases: ticks are different. Trends Parasitol. 2016;32:646-56.

3. Yusa A, Berry P, Cheng J, Ogden N, Bonsal BR, Stewart R, Waldick RC. Climate change, drought and human health in Canada. Int J Environ Res Public Health. 2015;12:8359-412.

4. Kulkarni MA, Berrang-Ford L, Buck PA, Drebot MA, Lindsay $\mathrm{LR}$, Ogden NH. Major emerging vector-borne zoonotic diseases of public health importance in Canada. Emerging Microbes \& Infection. 2015;4:e33. 